\title{
Gluon polarisation results from the COMPASS experiment
}

\author{
Luís Silva*† \\ LIP - Laboratório de Instrumentação e Física Experimental de Partículas \\ E-mail: lsilva@lip.pt
}

In the context of the nucleon spin structure, the contribution from the gluons plays an important role. The so-called gluon polarisation can be determined in DIS through the Photon-Gluon Fusion (PGF) process. In the COMPASS experiment, data were collected with a naturally polarised 160 $\mathrm{GeV} / c$ muon beam, impinging on a longitudinally polarised nucleon target. Two analyses aiming at the extraction of the gluon polarisation were performed. One is based on the identification of open charm events and the other on the selection of events with high- $p_{T}$ hadron pairs. The final results for the gluon polarisation from these analyses were obtained in LO approximation. For the open charm data an analysis in NLO was also performed. In the case of high- $p_{T}$ events, for the first time, the results are extracted in $3 x_{g}$ bins. Also $A^{2 h}$ asymmetries were recently evaluated in 2-dimensional bins of $x$ and $p_{T}^{2}$. In both analyses, a weighted method based on a neural network approach is used.

XXI International Workshop on Deep-Inelastic Scattering and Related Subject -DIS2013, 22-26 April 2013

Marseilles, France

\footnotetext{
*Speaker.

†n behalf of the COMPASs Collaboration.
} 


\section{Introduction}

The nucleon spin sum rule can be written in a heuristic way as: $\frac{1}{2}=\frac{1}{2} \Delta \Sigma+\Delta G+L_{q, g}$ [1], were $\Delta \Sigma$ and $\Delta G$ are the quark and gluon contributions to the nucleon spin, respectively, and $L_{q, g}$ are the partons orbital angular momentum. It was announced in the late 80 's and later confirmed by several experiments that the contribution carried by the quarks is $\sim 1 / 3$ of the nucleon spin [2-6]. The purpose of this work is to estimate the gluon polarisation $\Delta g / g$, which is deeply related with the gluon contribution to the nucleon spin. The data used in the present analyses were taken from the COMPASS experiment [7], where a naturally polarised $160 \mathrm{GeV} / \mathrm{c}$ muon beam scattering off polarised nucleons: deuterons, in the years 2002-2006, and protons in 2007.

\section{Gluon Polarisation Measurement}

The gluon polarisation is extracted using the so called Photon-Gluon-Fusion (PGF) process, depicted in Fig. 1 (c), in which the virtual photon interacts with a gluon coming from the nucleon via a quark, producing a $q \bar{q}$-pair. In order to measure the gluon polarisation, the longitudinal spin asymmetry $A_{\mathrm{LL}}$, defined as $\frac{\sigma^{\leftrightarrows}-\sigma^{\xi}}{\sigma^{\leftrightarrows}+\sigma^{\xi}}$, is used. Here $(\leftleftarrows)$ and $(\leftrightarrows)$ refer to the parallel and anti-parallel spin helicity configurations of the beam lepton $(\leftarrow)$ with respect to the target nucleon $(\Leftarrow$ or $\Rightarrow)$. In the analysis procedure, the raw experimental longitudinal spin asymmetry is extracted using the number of selected events measured in the two aforementioned beam-target configurations. The PGF process can be measured using two analysis methods: (i) selecting high $p_{T}$ hadron pair events, or (ii) selecting events containing open charm mesons.

\subsection{High- $p_{T}$ Analysis}

In the high $p_{T}$ analysis, the longitudinal spin asymmetry is calculated by selecting events containing high $p_{T}$ hadron pairs, i.e. with transverse momentum with respect the virtual photon direction above 0.7 and $0.4 \mathrm{GeV} / c$, for the highest and the second highest $p_{T}$ hadron respectively. A cut on $Q^{2}>1(\mathrm{GeV} / c)^{2}$ was also applied in order to select DIS events. Two other processes compete with the PGF process, the virtual photo-absorption leading order process (LP) and the gluon radiation (QCD Compton) process, illustrated in Fig.1 (a) and 1 (b),

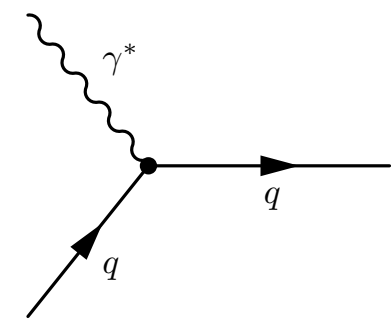

a)

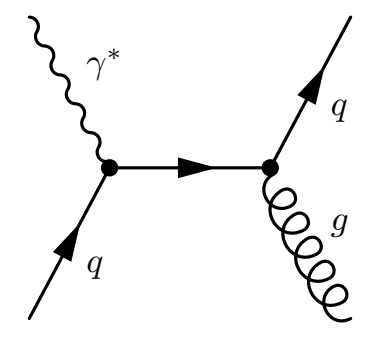

b)

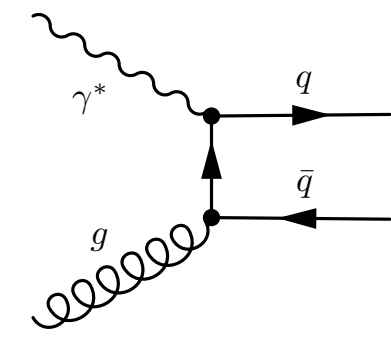

c)

Figure 1: DIS Feynman diagrams for $\gamma^{\star} N$ scattering: a) virtual photo-absorption (LP), b) gluon radiation (QCD Compton) and c) photon-gluon fusion (PGF). 
The longitudinal spin asymmetry for the high $p_{T}$ hadron pair data sample can thus be schematically written as:

$$
A_{\mathrm{LL}}^{2 h}\left(x_{B j}\right)=R_{\mathrm{PGF}} a_{\mathrm{LL}}^{\mathrm{PGF}} \frac{\Delta g}{g}\left(x_{g}\right)+R_{\mathrm{LP}} D A_{1}^{\mathrm{LO}}\left(x_{B j}\right)+R_{\mathrm{QCDC}} a_{\mathrm{LL}}^{\mathrm{QCDC}} A_{1}^{\mathrm{LO}}\left(x_{C}\right) .
$$

The process fractions are represented by $R_{i}, i$ referring to the different processes. The terms $a_{\mathrm{LL}}^{i}$ represent the partonic cross section asymmetries, $\Delta \hat{\sigma}^{i} / \hat{\sigma}^{i}$, also known as analysing power. The depolarisation factor $D$ is the fraction of the muon beam polarisation transferred to the virtual photon. $A_{1}^{\mathrm{LO}}$ is the virtual photon asymmetry. An equation similar to (2.1) can be written to express the inclusive asymmetry of a data sample, $A_{\mathrm{LL}}^{\text {incl }}$. Using eq. (2.1) for the high $p_{T}$ hadron pair sample and the same equation but for an inclusive sample, the final expression to extract the gluon polarisation is obtained:

$$
\frac{\Delta g}{g}\left(x_{g}^{a v}\right)=\frac{A_{\mathrm{LL}}^{2 h}\left(x_{B j}\right)+A^{\text {corr }}}{\lambda} .
$$

This formula corresponds to the longitudinal spin asymmetry $A_{\mathrm{LL}}^{2 h}$, measured directly from data, plus a correcting asymmetry $A^{\text {corr }}$ involving mainly the other two processes. The $\lambda$ factor contains the partonic asymmetries and the fractions of the involved processes. The partonic asymmetries and the process fractions need to be estimated using a dedicated and well tuned Monte Carlo (MC) simulation [8]. Details about this analysis can be found in Ref. [9]. A similar analysis was performed for the $Q^{2}<1(\mathrm{GeV} / c)^{2}$ data. This separation is due to the physical processes contained in the two $Q^{2}$ regimes. The $Q^{2}<1(\mathrm{GeV} / c)^{2}$ regime represents the quasi-real photon, in such conditions the photon may exhibit some inner structure. Therefore beside the three aforementioned processes the photon structure processes need to be included in the MC simulation. Details of this analysis can be found in Ref. [10].

\subsection{Open Charm Analysis}

In the case of the open charm analysis, the longitudinal spin asymmetries are calculated using data containing $D^{0}$ mesons. These events are identified by the invariant mass of the meson decay products, in particular $K \pi$ pairs. Applying a set of kinematic cuts, the combinatorial background is reduced. In addition the background is even more suppressed by tagging the $D^{0}$ candidates with a parent $D^{\star}$ using the $D^{\star} \rightarrow D^{0} \pi_{\text {slow }}$ channel. In this way three additional channels were included in the final analysis, $D^{0}$ to $K_{\text {sub }} \pi, K \pi \pi^{0}$ and $K \pi \pi \pi$. The former includes kaon candidates not identified by the RICH detector [7].

The number of events with $D^{0}$ particles in the final state is related to the gluon polarisation as shown by this expression:

$$
N_{t}=\alpha(S+B)\left[1+\beta\left(a_{\mathrm{LL}} \frac{S}{S+B} \frac{\Delta g}{g}+D \frac{B}{S+B} A^{\mathrm{bg}}\right)\right] .
$$

The subscript $t$ at the number of events stands for the muon-target spin configurations. The $\alpha$ factor contains the acceptance, muon flux and number of nucleons, and $\beta$ the beam and target polarisations and dilution factor. $S$ and $B$ represent the number of signal and background events taken under the invariant mass spectrum peak. $\frac{S(B)}{S+B}$ is the signal (background) purity. $A^{\text {bg }}$ is the asymmetry of the combinatorial background. Taking into account all the possible muon-target spin 
configurations, a set of equations is derived from eq. (2.3). Still, to solve this system of equations, the partonic asymmetry $a_{\mathrm{LL}}$ and the signal purity $\frac{S}{S+B}$ must be estimated. To compute the partonic asymmetry $a_{\mathrm{LL}}$ a dedicated MC simulation is used. A neural network approach is designed to parameterise the partonic asymmetry and the signal significance $\frac{S}{S+B}$. In the NLO analysis, QCD virtual and gluon bremsstrahlung corrections were included in the calculation of the analysing power for the PGF process, as well as the contribution from background physics processes. Details about this analysis can be found in Ref. [11].

\section{Results}

The results on the gluon polarisation in the nucleon, $\Delta g / g$, using the high $p_{T}\left(Q^{2}<1\right.$ and $Q^{2}>$ $1(\mathrm{GeV} / c)^{2}$, LO QCD order) and open charm (LO and NLO QCD order) analyses are presented here. In high $p_{T}$ analysis, at $Q^{2}>1(\mathrm{GeV} / c)^{2}$ the $\Delta g / g$ value found to be equal to $\Delta g / g=$ $0.125 \pm 0.060_{\text {stat }} \pm 0.063_{\text {sys }}$, at $x_{g}=0.09_{-0.04}^{+0.08}$ and with a hard scale of $\left\langle\mu^{2}\right\rangle=3(\mathrm{GeV} / c)^{2}$. This measurement is presented in Tab. 1 in three statistically independent points. The analysis of the $Q^{2}<1(\mathrm{GeV} / c)^{2}$ data gives $\Delta g / g=0.016 \pm 0.058_{\text {stat }} \pm 0.055_{\text {sys }}[10]$.

\begin{tabular}{llll}
\hline & $1^{s t}$ Bin & $2^{\text {nd }}$ Bin & $3^{r d}$ Bin \\
\hline$\Delta g / g$ & $0.147 \pm 0.091 \pm 0.088$ & $0.079 \pm 0.096 \pm 0.082$ & $0.185 \pm 0.165 \pm 0.143$ \\
$x_{g}^{a v}$ & $0.07_{-0.03}^{+0.05}$ & $0.10_{-0.04}^{+0.07}$ & $0.17_{-0.06}^{+0.10}$ \\
\hline
\end{tabular}

Table 1: Gluon polarisation results in bins of $x_{g}$.

Additionally, using the same data sample entering this analysis, i.e. with the same event selection used for the $\Delta g / g$ analysis, the asymmetries were calculated in a 2-dimensional $5 \times 5$ binning in $x_{B j}$ and $\sum p_{T}^{2}$. The table with the results is available in [9].

The gluon polarisation value for the open charm LO QCD analysis was found as $\Delta g / g=$ $-0.06 \pm 0.21_{\text {stat }} \pm 0.08_{\text {sys }}$ at $x_{g}=0.11_{-0.05}^{+0.11}$ and $\Delta g / g=-0.13 \pm 0.15_{\text {stat }} \pm 0.15_{\text {sys }}$ at $x_{g}=0.20_{-0.08}^{+0.13}$ for NLO QCD order. Both results are given at a hard scale of $\left\langle\mu^{2}\right\rangle=13(\mathrm{GeV} / c)^{2}$. All the gluon polarisation results of the COMPASS Collaboration [9-11] are summarised in Fig. 2 together with the SMC [12] and HERMES [13] results.
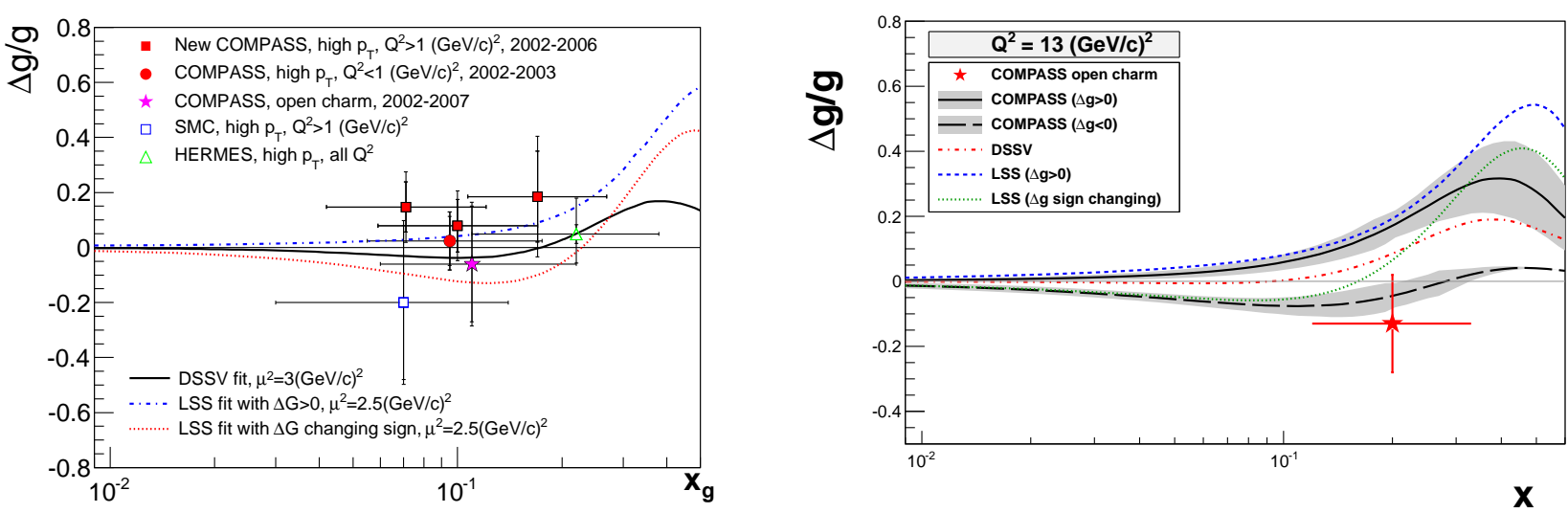

Figure 2: Gluon polarisation results: LO QCD order results (left side plot), NLO QCD order results (right side plot) and also theoretical fits form the DSSV [14] and LSS [15]. 


\section{Conclusions}

The gluon polarisation results presented here are compatible with those from the previous analyses, and indicate small values of $\Delta g / g$ in the region of $x_{g} \simeq 0.1$. These results as also confirmed by the NLO QCD global analysis fit curves [14, 15], which also predicts a small value for the gluon spin contribution to the nucleon.

\section{References}

[1] R. L. Jaffe, A. Manohar, The g(1) Problem: Fact and Fantasy on the Spin of the Proton, Nucl. Phys. B337 509-546.

[2] J. Ashman, et al., A measurement of the spin asymmetry and determination of the structure function g(1) in deep inelastic muon proton scattering, Phys. Lett. B 206364.

[3] K. Abe, et al., Measurements of the proton and deuteron spin structure functions g1 and g2, Phys. Rev. D 58, 112003 [hep-ph/9802357].

[4] P. Anthony, et al., Deep Inelastic Scattering of Polarized Electrons by Polarized ${ }^{3}$ He and the Study of the Neutron Spin Structure, Phys. Rev. D 546620 [hep-ex/9610007].

[5] D. Adams, et al., Spin structure of the proton from polarized inclusive deep-inelastic muon proton scattering, Phys. Rev. D 565330 [hep-ex/9702005].

[6] A. Airapetian, et al., Measurement of the proton spin structure function gl( $p$ ) with a pure hydrogen target, Phys. Lett. B 442484 [hep-ex/9807015].

[7] B. Abbon, et al., The Compass Experiment at CERN, Nucl. Instrum. Meth. A 577 455-518 [hep-ex/0703049].

[8] L. Silva, Measurement of the gluon polarisation through high- $p_{T}$ hadron production in COMPASS, PhD Thesis, IST, Univ. Técnica de Lisboa, 2011.

[9] C. Adolph, et al., Leading order determination of the gluon polarisation from DIS events with high- $p_{T}$ hadron pairs, Phys. Lett. B 718922 [hep-ex/1202.4064].

[10] E. Ageev, et al., Gluon polarization in the nucleon from quasi-real photoproduction of high-p(T) hadron pairs, Phys. Lett. B 633 25-32 [hep-ex/0511028].

[11] C. Adolph, et al., Leading and next-to-leading order gluon polarization in the nucleon and longitudinal double spin asymmetries from open charm muoproduction, Phys. Rev. D 87052018 [hep-ex/1211.6849].

[12] B. Adeva, et al., Spin asymmetries for events with high $p(T)$ hadrons in DIS and an evaluation of the gluon polarization Phys. Rev. D D 70012002 [hep-ex/ 0402010 ].

[13] A. Airapetian, et al., Measurement of the spin asymmetry in the photoproduction of pairs of high $p(T)$ hadrons at HERMES, Phys. Rev. Lett. 84 2584-8 [hep-ex/9907020].

[14] E. Leader, A. Sidorov and D. Stamenov, Determination of Polarized PDFs from a QCD Analysis of Inclusive and Semi-inclusive Deep Inelastic Scattering Data, Phys. Rev. D 82 114018-29.

[15] D. de Florian, R. Sassot, M. Stratmann and W. Vogelsang, Extraction of Spin-Dependent Parton Densities and Their Uncertainties, Phys. Rev. D 80 034030-55. 